\title{
A New Face Recognition Technique using Gabor Wavelet Transform with Back Propagation Neural Network
}

\author{
V. Balamurugan \\ Phd,Professor \& Head-PG \\ Department of ECE \\ Nehru Institute of Engineering \\ \&Technology \\ Coimbatore-641105, India.
}

\author{
Mukundhan Srinivasan \\ Pre - Final Year \\ Department of ECE \\ Alpha College of Engineering \\ Chennai-600124, India.
}

\author{
Vijayanarayanan.A \\ Pre - Final Year \\ Department of ECE \\ Alpha College of Engineering \\ Chennai-600124, India.
}

\begin{abstract}
This paper introduces a new face recognition technique using Gabor Wavelet transform and Back propagation network. Face recognition being regarded as a fundamental technology of biometrics has been applied to a variety of areas, including computer vision and pattern recognition. In this proposed approach, the features of the query face image and database face images have been extracted using Gabor transform and trained using BPN. The main objective of this proposed system is to develop an efficient face recognition system by improving the efficiency of the existing face recognition systems. The proposed system has been developed to provide efficiency in terms of retrieval accuracy and precision. The precision improved by $100 \%$ and average recall rate of up to $97 \%$ for the database of 100 images. The efficiency of the proposed system obtained as $100 \%$.
\end{abstract}

\section{General Terms}

Face Recognition, Wavelet Transform, Neural Network, Feature extraction

\section{Keywords}

Gabor Wavelet transforms (GWT), back propagation Network (BPN), eigenface.

\section{INTRODUCTION}

Face recognition has traditionally been performed with facial feature extracted from two dimensional images. The features are placed in sets, and decision boundaries are created by which input images may be classified and identified. However, there are two methods face recognition procedure in general, global characteristics and local feature characteristics. A successful face recognition methodology depends heavily on the particular choice of the features used by the (pattern) classifier [4]. Feature selection in pattern recognition involves the derivation of salient features from the raw input data in order to reduce the amount of data used for classification. The most representative recognition techniques frequently used in conjunction with face recognition are principal component analysis (PCA) [1], independent component analysis (ICA) [2], and Fisher's linear discriminant analysis (LDA) [3]. The PCA approach is also known as eigenface method which is a popularly unsupervised statistical technique that supports finding useful image representations. It also exhibits optimality when it comes to dimensionality reduction.

However, PCA is not ideal for classification purposes mainly because of the fact that it retains unwanted variations occurring due to lighting and facial expression [3]. There are numerous extensions to the standard PCA method [4]-[8]. Meanwhile, the LDA method, also known as Fisher's linear discriminant face method, is a supervised learning approach whose functioning depends on class-specific information. This statistically motivated method maximizes the ratio of between class scatter and within-class scatter and is also an example of a class-specific learning method. Again, there are various enhancements made to the LDA [9]-[14].

On the other hand, while PCA-based and LDA-based approaches depend only on second-order statistical structure between pixels in the face image, ICA method has been used to find statistically independent basis images or coefficient for the face images to deal with the sensitivity to higher order image statistics [2]. In particular, the ICA technique is related to the blind source separation (BSS) problem, where the goal is to decompose an observed signal into a linear combination of several unknown independent signals. This method has demonstrated to be successful in various fields such as speech recognition [15], three-dimensional (3-D) object recognition , natural images , unsupervised classification [9], bioinformatics, texture segmentation , electroencephalogram (EEG)[15], functional magnetic resonance imaging (f-MRI) signals [9] to name a few representative examples. In the area of face recognition, Bartlett [2] first developed facerecognition system with two different architectures based on the principle of optimal information transfer (InfoMax) proposed by Bell and Sejnowski.

The purpose of these two architectures is to find statistically independent basis images or factorial face code. The results clearly showed that both ICA representations were superior to the representations based on the PCA. Meanwhile, Moghaddam [9] used two different algorithms with the first architecture based on fourth-order cumulates.

Moghaddam [9] found that the ICA is not unique in comparison with the PCA due to the variety of different techniques used to compute the basis and iterative optimization schemes being involved there. In order to analyze such contradictory claims, Draper [9] performed a thorough comparison of the two techniques.

However, The aforementioned techniques unable to recognize the faces with $100 \%$ precision but our proposed approach combined with Gabor features with Back propagation neural (BPN) network capable to achieve best results.

\section{PROPOSED ARCHITECTURE}

The architecture developed for feature based face recognition is illustrated schematically in Figure1.The face feature extraction and face recognition is the main contribution of the architecture. The query face is processed to compute 
features, the distance between the two eyes and nose as triangle. There are three main features have been used in our system. The two eyes and nose are feature in our proposed system. The two distances between the two eyes and nose have been computed for query face image. The features are of the computed query face and similar features of the group photo of 59 persons are equal then the face recognized in the group photo.

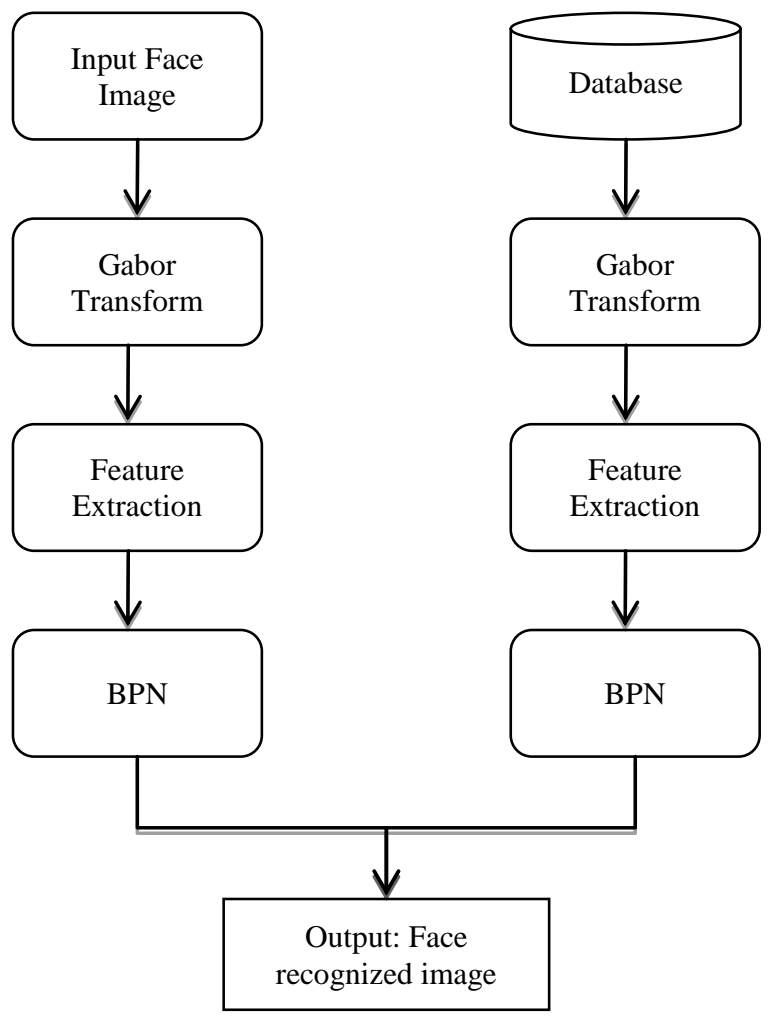

Figure 1. Proposed face recognition system

The output faces are detected similar to the feature values of the query image. The query face input image has been given to Gabor transform and the three features are extracted. These features are trained using BPN. The proposed system able to recognize the same features in the group photo consists of 59 faces. The query face and identified by the face exactly matches. Quadratic distance measure is used for comparison. Similar feature face images are displayed. The exact output face images as relevant to query face image is recognized.

\section{GABOR WAVELET TRANSFORM}

\section{(GWT)}

The processing of facial images by Gabor filter is chosen for its biological relevance and technical properties. The Gabor filter kernels have similar shapes as the receptive fields of simple cells in the primary visual cortex have been set.

Wavelet representation shown in equation 1 is especially useful for feature analysis in vision problems because its theory provides powerful properties, such as algorithms for dominant point's detection, and local periodic pattern analysis. In fact wavelet constitutes an efficient mathematical tool for the detection and characterization of signal singularities [3] [6].

$$
\mathbf{y}[\mathbf{n}]=(\boldsymbol{x} * \boldsymbol{g})[\boldsymbol{n}] \sum_{\boldsymbol{k}=-\infty}^{\infty} \mathbf{x}[\mathbf{k}] \mathrm{g}[\mathbf{n}-\mathbf{k}]
$$

Multi-resolution analysis techniques decompose the signal into components at different scales so that the coarsest scale components carry the global approximation information while the finer scale components contain the details information. Additionally some recent psychophysics models suggest that the human visual system processes and analyses image information at different resolutions.

2D-DWT is a process which decomposes a signal, that is a series of digital samples, by passing it through two filters, a low pass filter $\mathrm{L}$ (equation 2) and high pass filter $\mathrm{H}$ (equation 3 ). The low pass sub band represents a down sampled version of the original signal. The high pass sub band represents residual information of the original signal, needed for the perfect reconstruction of the original set from the low resolution.

$$
\begin{aligned}
& y_{\text {low }}[\mathrm{n}]=\sum_{k=-\infty}^{\infty} \mathrm{x}[\mathrm{k}] \mathrm{g}[2 \mathrm{n}-\mathrm{k}] \\
& y_{\text {high }}[\mathrm{n}]=\sum_{k=-\infty}^{\infty} \mathrm{x}[\mathrm{k}] \mathrm{h}[2 \mathrm{n}-\mathrm{k}]
\end{aligned}
$$

\section{NEURAL NETWORK}

Neural network is used as an adaptive retrieval system which incorporates learning capability into the network module where the network weights represent adaptability. This learning approach has several advantages over traditional retrieval approaches.

The proposed system is based on Back propagation neural (BPN) network. There are three layers of BPN network including input layer, hidden layer and output layer shown in Figure 2.The input layer propagates the feature vectors to each node in the middle layer. Middle layer nodes compute output values which given to the output layer. The output layer nodes compute the network output for the particular feature vector is shown in Figure 3

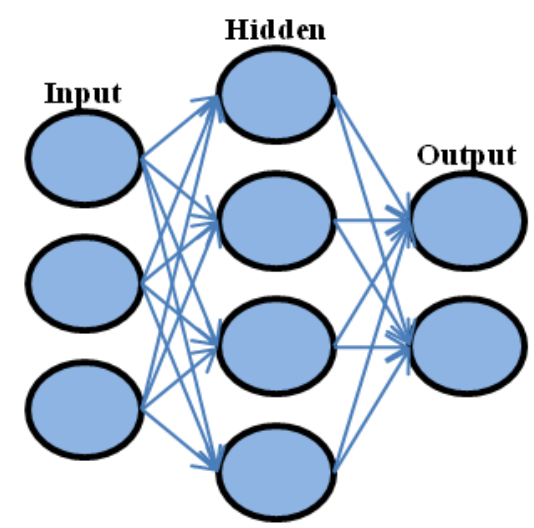

Figure 2. Three layered BPN

Since the network weights are initialized to random values, it is unlikely that reasonable outputs will result before training. The weights are adjusted to reduce the error by propagating the output error backward through the network. Compute the error values for each node in the output layer. Compute the error for the middle layer nodes. This is done by attributing a portion of the error due to the middle layer node which feed that output node. The amount of error due to middle layer 
node depends upon size of the weight assigned to the connection between the two nodes. Adjust the weight values

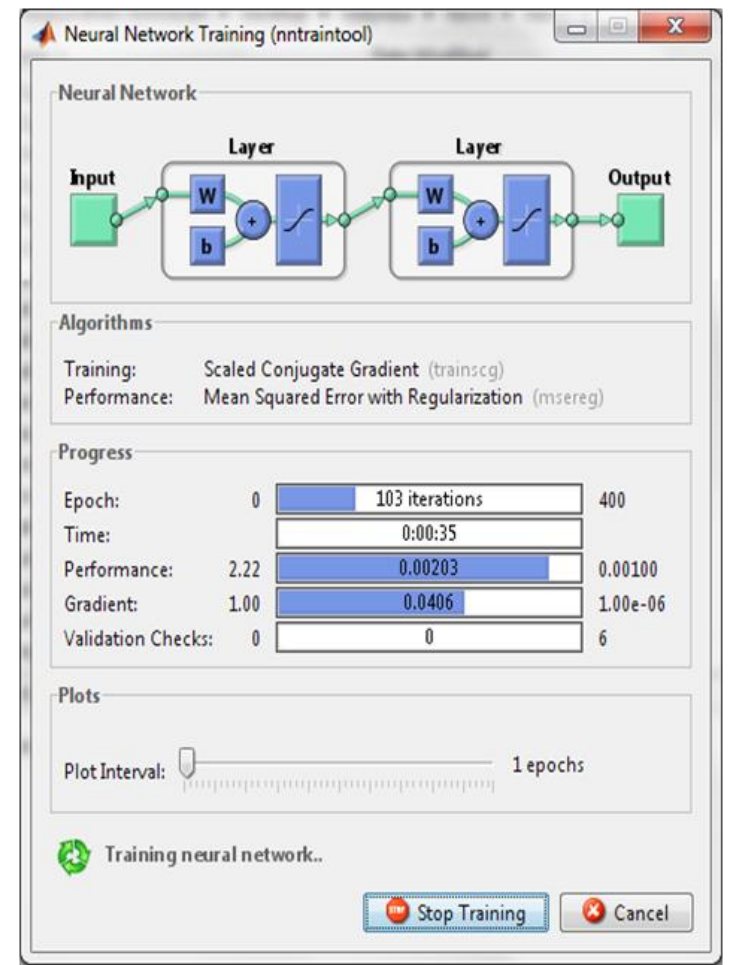

to

Figure 3. BPN Training

improve network performance using delta rule.

\subsection{BPN TRAINING ALGORITHM}

1. Training features to the neural network.

2. Compare the network's output to the desired output from that features.

3. Calculate the error in each output neuron.

4. For each neuron, calculate what the output of each feature vector and the output must be adjusted to match the desired output.

5. Adjust the weights of each neuron to lower the local error.

6. Repeat from step 3 on the neurons at the previous level until the output is obtained.

The performance plot for the BPN is shown in Figure 4. The performance plot clearly states the optimum is reached at 93 epochs. The Training plot is shown in Figure 5.

\subsection{NEURAL NETWORK PARAMETERS}

Neural network used in our proposed approach is BPN for learning; its parameters shown in Figure 4 to Figure 8. The network performance plot shown in Figure is 4. The network Training State plot is shown in Figure 5. The network regression plot is shown in Figure 6. The network Fit plot is

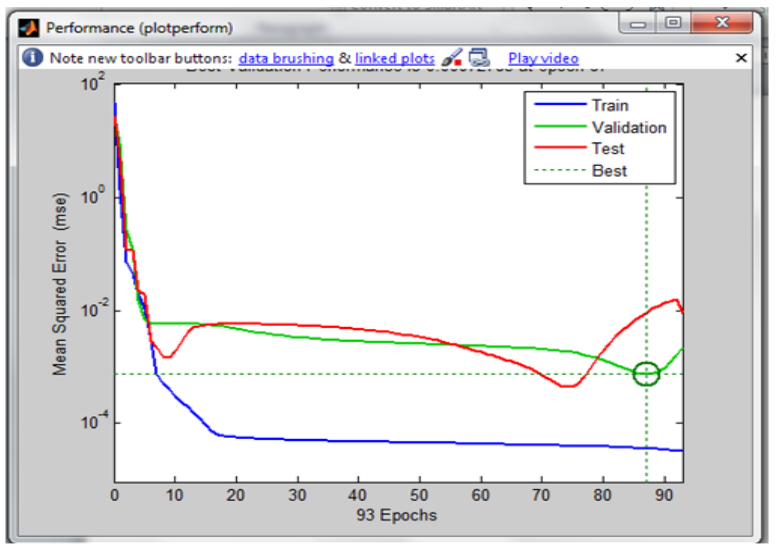

shown in Figure 7.

The function plot perform (TR) plots the training, validation, and test performances given the training record TR returned by the function train as shown in Figure 4 .

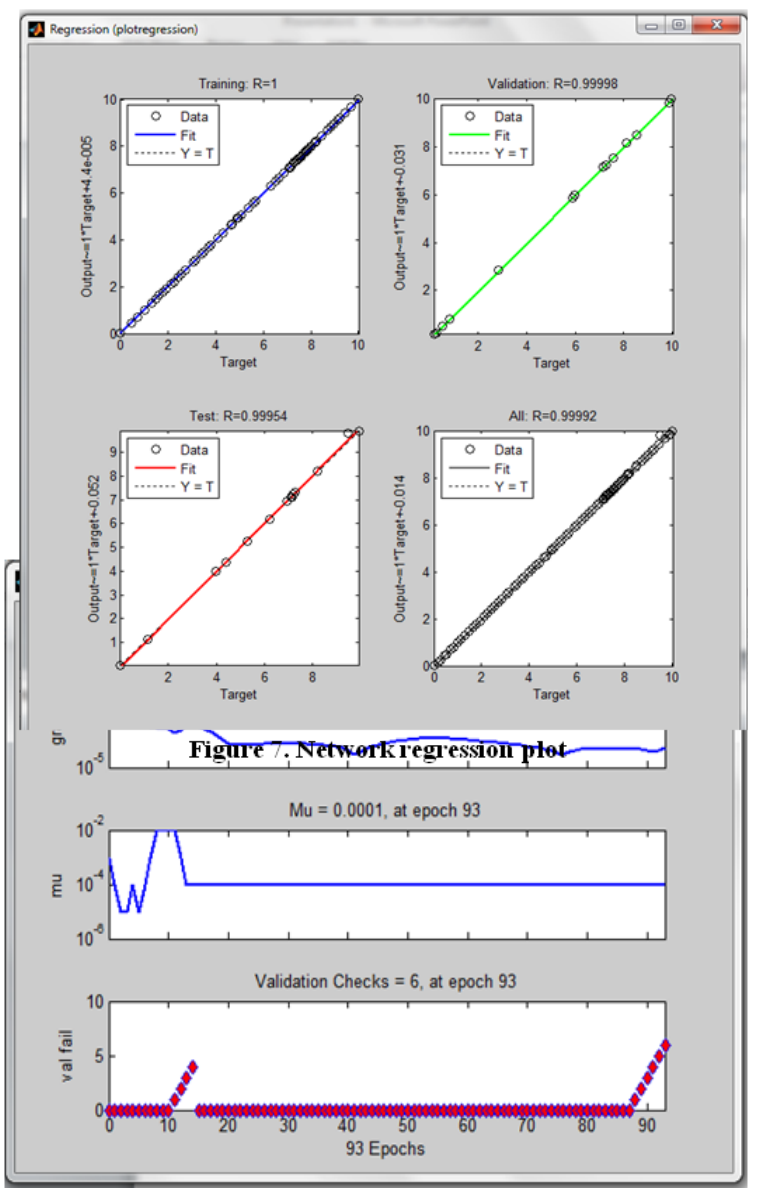

Figure 6. Network training state plot

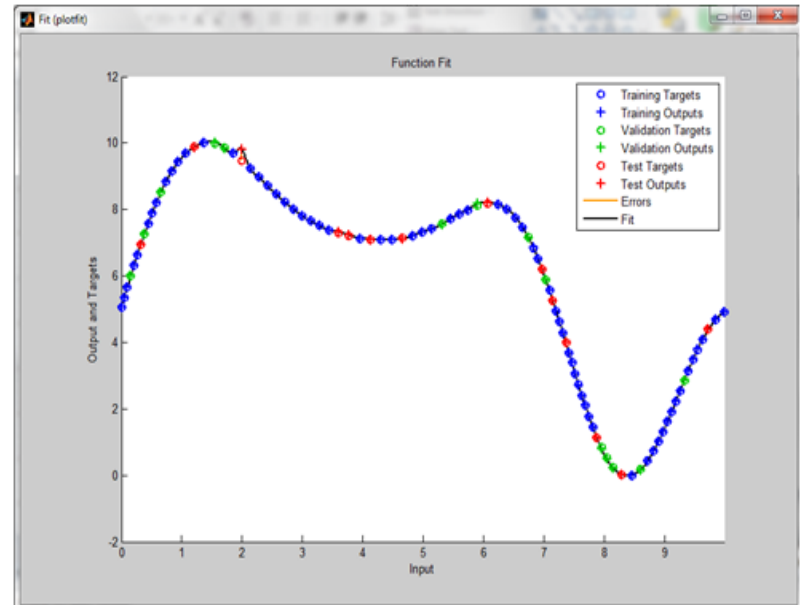

Figure 8. Network Fit plot

\section{EXPERMENTAL RESULTS}

The experiments were conducted using an Intel i7 processor at $3.40 \mathrm{GHz}$ with MATLAB R2010b. The proposed framework is tested with a database of about 100 faces to detect the 59 
faces of 47 final year students of Department of Electronics and Communication Engineering and 12 faculty members from the Alpha College of Engineering shown in the group photo in Figure 9. In current experiment performed on the group photo of with 59 face images from total faces 100 face images, feature extraction with threshold has been simulated and shown in Figure 10. Figure 11 shows the background removing using edge detection. Figure 12 shows the proposed approach face recognition technique. Figure 13 shows the some of the sample face images used for our experiment. To measure the performance of the proposed system, average recall rate (AVRR), precision are used.

\subsection{EDGE DETECTION}

The edge detection used in our proposed method is Sobel edge detection technique. It is a discrete differentiation operator, computing an approximation of the gradient of the image intensity function. At each point in the image, the result of the Sobel operator is either the corresponding gradient vector or the norm of this vector. The Sobel operator is based on convolving the image with a small, separable, and integer valued filter in horizontal and vertical direction and is therefore relatively inexpensive in terms of computations.

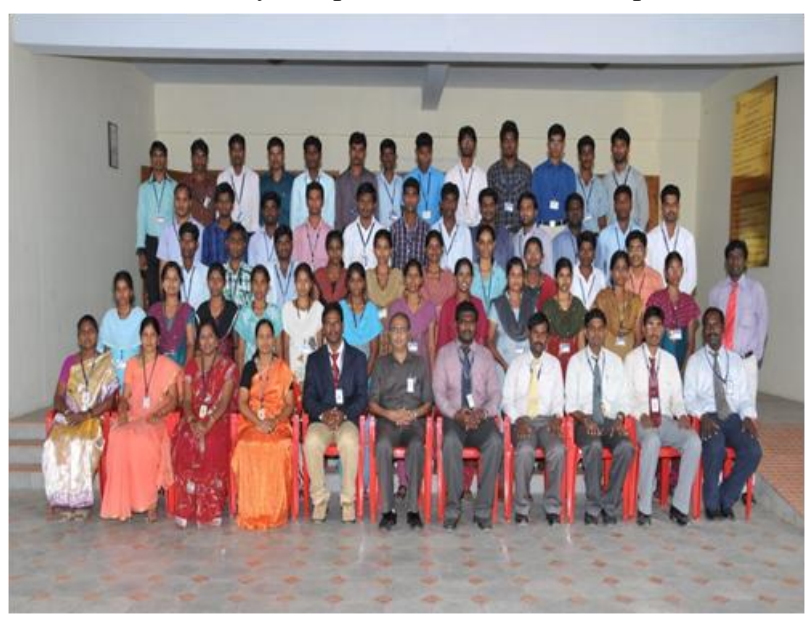

Figure 9. Input image

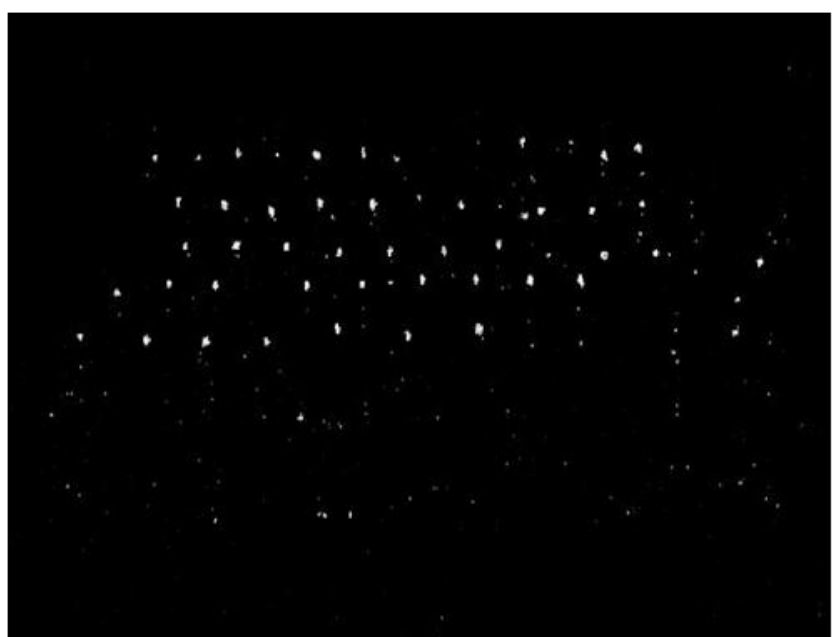

Figure 11. Background removed using Sobel edge detection

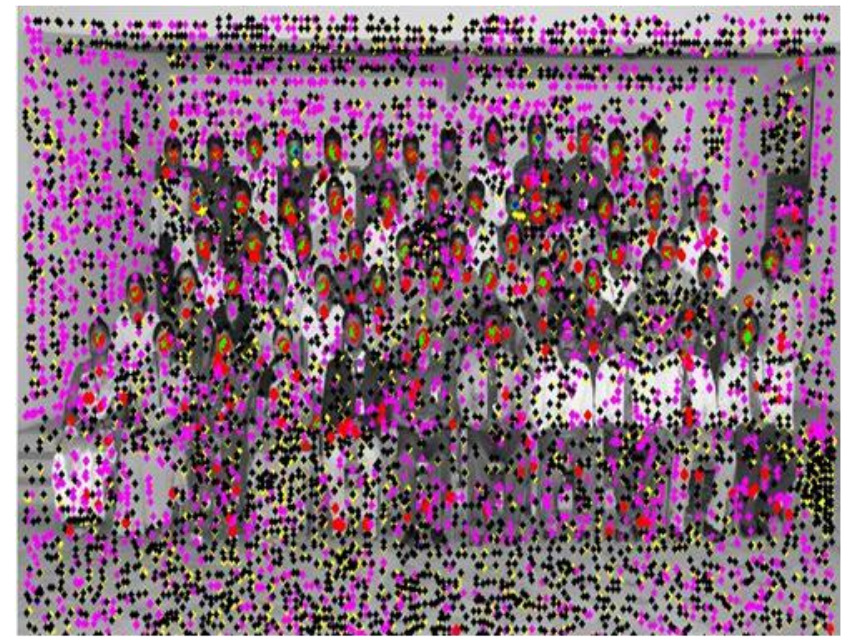

Figure 10. Feature extraction with threshold

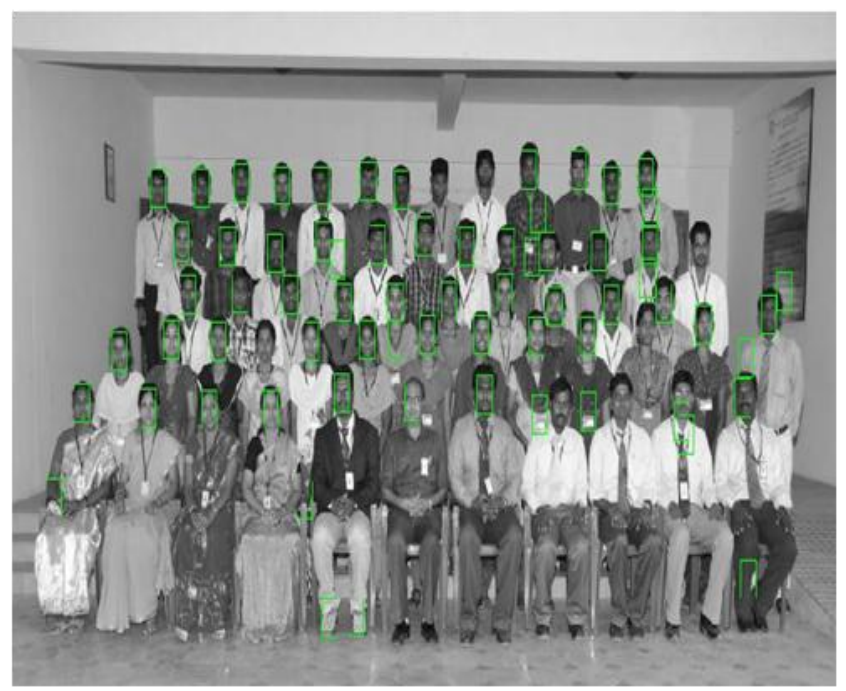

Figure 12. Simulated output image after recognition
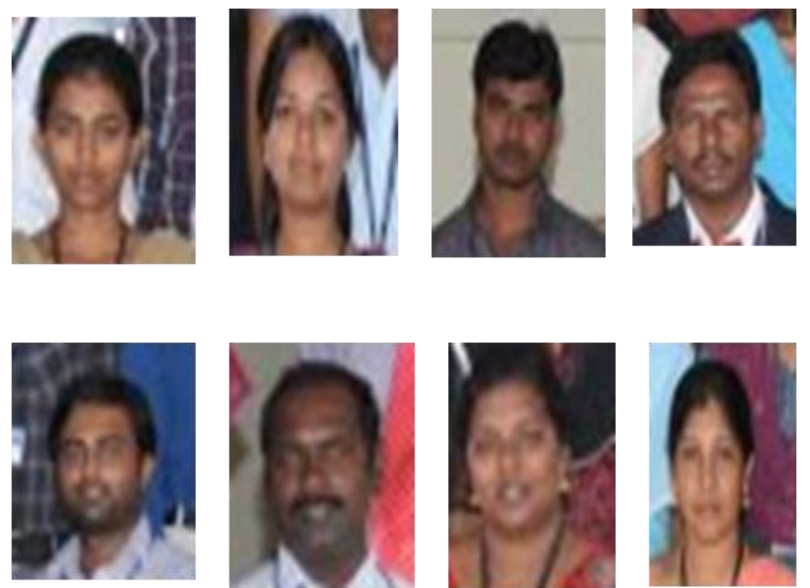

Figure 13. Example faces 


\section{SIMULATION RESULTS}

Table 1. Successful recognition rates and \% Precision

\begin{tabular}{|c|c|c|c|}
\hline $\begin{array}{c}\text { No. of } \\
\text { Face } \\
\text { Images }\end{array}$ & $\begin{array}{c}\text { Successfully } \\
\text { recognized }\end{array}$ & $\begin{array}{c}\text { Unsuccessful } \\
\text { recognition }\end{array}$ & $\begin{array}{c}\text { Percentage } \\
\text { Precision (\%) }\end{array}$ \\
\hline 10 & 10 & - & 100.00 \\
\hline 20 & 18 & - & 100.00 \\
\hline 30 & 29 & - & 100.00 \\
\hline 40 & 40 & - & 100.00 \\
\hline 50 & 48 & - & 100.00 \\
\hline 75 & 74 & - & 100.00 \\
\hline 100 & 98 & - & 100.00 \\
\hline
\end{tabular}

Table 1 shows the successful recognition rates and \% precision obtained for the 100 queries considered. Our proposed approach yields up to $97.045 \%$.

Table 2 shows the Successful recognition rates and \% Average recall rate obtained for the 100 queries considered. Our proposed approach gives 100\% efficiency.

The efficiency of our proposed method is shown in figure 14 which is a plotted between Number of eigenfaces and the Recognition Rate. The plot shows that the proposed method is much more efficient than the other existing methods.

Table 2. Successful recognition rates and \% Average recall

\begin{tabular}{|c|c|c|c|}
\hline $\begin{array}{c}\text { No. of } \\
\text { Face } \\
\text { Images }\end{array}$ & $\begin{array}{c}\text { Successfully } \\
\text { recognized }\end{array}$ & $\begin{array}{c}\text { Unsuccessful } \\
\text { recognition }\end{array}$ & $\begin{array}{c}\text { \% Average } \\
\text { recall rate }\end{array}$ \\
\hline 10 & 10 & - & 100.00 \\
\hline 20 & 18 & - & 100.00 \\
\hline 30 & 29 & - & 100.00 \\
\hline 40 & 40 & - & 100.00 \\
\hline 50 & 48 & - & 100.00 \\
\hline 75 & 74 & - & 100.00 \\
\hline 100 & 98 & - & 100.00 \\
\hline
\end{tabular}

\section{CONCLUSION}

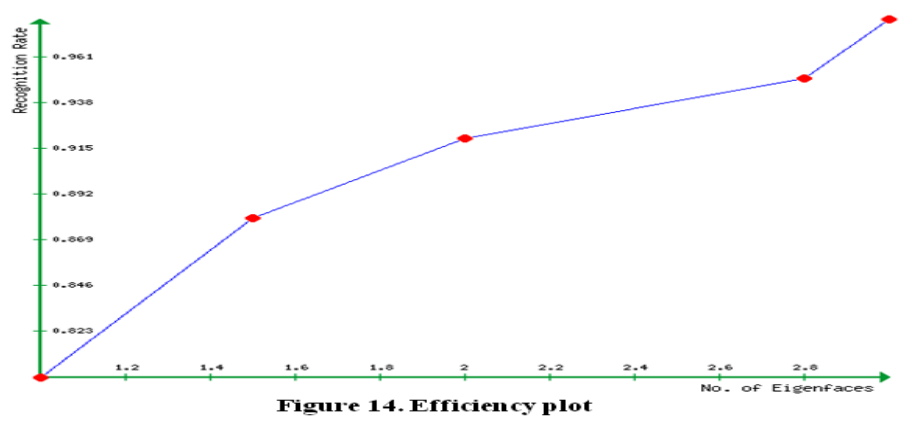

In this paper, we proposed a novel Face recognition technique using Gabor Wavelet Transform (GWT) and Back propagation Neural Network (BPN). Face recognition is a biometric which uses computer software like MATLAB to determine the identity of the individual. Face recognition falls into the category of biometrics which is using distinguishing traits. Face images from a database are compared with query face images. In this paper, we pursue a new approach in which similarity is learned from training examples over the neural network which is provided by human observers. The main contribution of this works is in building various features required by a working system for efficient and effective retrieval of images. This proposed approach greatly reduces user's effort and captures the user's information needed more precisely. Furthermore, the effectiveness of the proposed approach has been validated by a large number of experiments yielding $100 \%$ overall.

\section{REFERENCE}

[1] M. Turk and A. Pentland, "Face recognition using eigenfaces," in Proc.IEEE Conf. Comput. Vis. Pattern Recognit., 1991, pp. 586-591.

[2] M. S. Bartlett, J. R. Movellan, and T. J. Sejnowski, "Face recognition by independent component analysis," IEEE Trans. Neural Netw., vol.13, no. 6, pp. 1450-1464, Nov. 2002.

[3] P. N. Belhumeur, J. P. Hespanha, and D. J. Kriegman, "Eigenfaces vs.fisherfaces: Recognition using class specific linear projection," IEEE Trans. Pattern Anal. Mach. Intell., vol. 19, no. 7, pp. 711-720, Jul.1997.

[4] A. Pujol, J. Vitria, F. Lumbreras, and J. J. Villanueva, "Topological principal component analysis for face encoding and recognition," PatternRecognit. Lett., vol. 22, pp. 769-776, 2001.

[5] H. C. Kim, D. Kim, and S. Y. Bang, "Face recognition using the mixture-of-eigenfaces method," Pattern Recognit. Lett., vol. 23, pp.1549-1558, 2002

[6] K. I. Kim, K. Jung, and H. J. Kim, "Face recognition using kernel principal component analysis," IEEE Signal Process. Lett., vol. 9, no. 2, pp. 40-42, Feb. 2002.

[7] B. Li and Y. Liu, "When eigenfaces are combined with wavelets," Knowledge-Based Syst., vol. 15, pp. 343-347, 2002.

[8] R. Gottumukkal and V. K. Asari, "An improved face recognition technique based on modular PCA approach," Pattern Recognit. Lett., vol.24, pp. 429-436, 2004.

[9] Keun-Chang Kwak, Member, IEEE, and Witold Pedrycz, Fellow, IEEE, "Face Recognition Using an Enhanced Independent Component Analysis Approach." IEEE Transactions On Neural Networks, Vol. 18, No. 2,pp.530-541 MARCH 2007.

[10] H. Yu and J. Yang, "A direct LDA algorithm for highdimensional data with application to face recognition," Pattern Recognit., vol. 34, pp.2067-2070, 2001.

[11] Z. Jin, J. Y. Yang, Z. S. Hu, and Z. Lou, "Face recognition based on the uncorrelated discriminant transformation," Pattern Recognit., vol.34, pp. 14051416, 2001.

[12] K. C. Kwak and W. Pedrycz, "Face recognition using fuzzy integral and wavelet decomposition method," IEEE Trans. Syst., Man, Cybern.B, Cybern., vol. 4, no. 4 pp. 1666-1675, Aug. 2004. 
[13] L. Juwei, K. N. Plataniotis, and A. N. Venetsanopoulos, "Face recognition using kernel direct discriminant analysis algorithms," IEEE Trans.Neural Netw., vol. 14, no. 1, pp. 117-126, Jan. 2003.

[14] .C. Liu and H. Wechsler, "Gabor feature based classification using the enhanced Fisher linear discriminant model for face recognition," IEEETrans. Image Process., vol. 11, no. 4, pp. 467-476, Apr. 2002.

[15] J.Yang, A. F. Frangi, and J. Y.Yang, “Anewkernel Fisher discriminant algorithm with application to face recognition," Neurocomput., vol. 56, pp. 415-421, 2004.

[16] Gilbert Strang, MIT. Linear algebra and its applications. Academic Press (1976), Second edition: Harcourt Brace Jovanovich (1980), Third edition: Brooks/Cole (1988), Fourth edition: Brooks/Cole (2005).

[17] I. Biederman. Recognition by components: A theory of human image understanding. Psych Rev, vol. 94, pp.115$147,1987$.
[18] I. Rock. The logic of perception. Cambridge, MA: MIT Press, 1983.

[19] V. Brian, Funt, Kobus Barnard, Lindsay Martin. Is machine colour constancy good enough? Proceedings of the 5th European Conference on Computer Vision, vol. 1, pp.445-459, June 02-06, 1998.

[20] S. Zeki. A vision of the brain. Blackwell Scientific Publications Oxford, 1993.

[21] J. Ingemar, Cox, Joumana Ghosn, N. Peter, Yianilos. Feature-based face recognition using mixture-distance. Proceedings of the 1996 Conference on Computer Vision and Pattern Recognition (CVPR '96), pp.209, June 1820, 1996

[22] V. Govindaraju, R. K. Krishnamurthy. Holistic handwritten word recognition using temporal features derived from off-line images, Pattern Recognition Letters, Elsevier Science, vol. 17(5), pp.537-540(4), May $1,1996$. 